\title{
Genetic Variation among Sweetpotatoes Propagated through Nodal and Adventitious Sprouts
}

\author{
Arthur Q. Villordon ${ }^{1}$ and Don R. LaBonte ${ }^{2}$ \\ Department of Horticulture, Louisiana Agricultural Experiment Station, Louisiana State University \\ Agricultural Center, Baton Rouge, LA 70803
}

Additional index words. Ipomoea batatas, arbitrary primers, RAPD/AP-PCR, clonal variability, tissue culture

\begin{abstract}
Genetic uniformity was assessed among sweetpotato (Ipomoea batatas) clones propagated through adventitious and nodal procedures. A single sprout each of 'Jewel,' 'Sumor,' and L87-95 was used as source of clonal plants that were simultaneously propagated through conventional adventitious procedures and a tissue culture-based nodal culture technique. A sample of 15 decamer primers generated 64 scorable amplified fragments in a PCR-based assay, 29 of which were putatively polymorphic across $n=60$ samples ( 10 each of nodal and adventitiously derived plants/genotype). Within adventitiously derived materials, putative polymorphisms ranged from $4.7 \%$ to $31.3 \%$ depending on the genotypic class. In contrast, putative polymorphisms ranged from $0.0 \%$ to $3.1 \%$ among nodally derived samples. Marker loci differentiated genotypes as well as putative marker phenotype variants through a multidimensional scaling analysis of the genetic similarity matrix. An 'analysis of molecular variance' shows that genotypic effects accounted for $88.7 \%$ of the total molecular marker variability, while propagation effects (within genotypic groups) accounted for $11.3 \%$. Results confirm that clonal plants derived from preexisting meristematic regions are more genetically uniform than plants propagated from adventitious origins.
\end{abstract}

In sweetpotato, the adventitious sprouts that arise from storage roots are used as propagules. This vegetative propagation theoretically insures genetic fidelity, however, variability is still observed at the phenotypic level among propagules. Environmental interactions probably contribute to this variability, wherein the expression of quantitative traits is conditioned by prevailing environmental factors (Collins et al., 1987; Kannua and Floyd, 1988; Ngeve and Bouwkamp, 1993). Confounding effects arise due to phenotypic plasticity, e.g., several types of leaves occurring on individuals of most species (Yen, 1974) and variable root yield among hills within a location (Steinbauer et al., 1943). Even rigorously selected materials in foundation seed programs continue to exhibit qualitative changes such as root skin and flesh color anomalies (Sloan, 1994).

The possible influence of the method of propagation on phenotypic uniformity of sweetpotato has been previously investigated. Sweetpotato storage root yields differed between adventitiously derived plants and propagules obtained through vine cuttings (nodal) (Huett, 1982), the latter being the traditional propagation method in tropical production systems where plants grow in the field throughout the year. Yield differences between propagation methods varied from year to year, and this variability was attributed to factors such as nonuniform sprout size that confounded observed variability. Similarly, phenotypic variability was assessed among in vitro-derived nodal plants and adventitiously derived clonal plants (Templeton-Sommers and Collins, 1986). It

Received for publication 24 Mar. 1995. Accepted for publication 8 Sept. 1995. Approved for publication by the Director of the Louisiana Agricultural Experiment Station as manuscript no. 95-28-9134. The research was supported by funds from the Louisiana Methodist World Hunger Scholarship Program, the Louisiana Sweetpotato Advertising and Development Commission, and by state and matching funds allocated to the Louisiana State Univ. Agricultural Center. We acknowledge the help of Michael Stine, LSU School of Forestry, Wildlife, and Fisheries, for access to the NTSYS-pc software used for molecular marker analysis. We also thank Thomas Kubisiak, former graduate student in LSU SFWF, for technical suggestions. The cost of publishing this paper was defrayed in part by the payment of page charges. Under postal regulations, this paper therefore must be hereby marked advertisement solely to indicate this fact.

${ }^{1}$ Graduate student.

${ }^{2}$ Associate professor. was observed that in vitro-derived plants were significantly more uniform with lower root skin and flesh color mutations than adventitiously derived plants. This study indicated that the method of propagation influenced phenotypic variability, but it was unknown whether mutations, phenotypic variability, or epigenetic changes were contributing to this variation.

Adventitious buds of sweetpotato develop from callus, wound periderm, vascular cambium, or anomalous cambium (Edmond and Ammerman, 1971; Esau, 1977; Fahn, 1982). Such buds are derived from somatic cells and are possibly genetically variant (De Klerk, 1990). In contrast, preexisting meristems, e.g., sweetpotato nodes, develop into true-to-type plants whereby little if any genetic variability is presumed to occur.

The primary objective of this study was to assess the extent of genomic stability among sweetpotato plants obtained from nodal and adventitious sprouts. Molecular markers, such as those generated through the use of arbitrary primers, RAPD/AP-PCR (Welsh and McClelland, 1990; Williams et al., 1990), provide tests of genetic uniformity that are most likely independent of the confounding effects of the environment.

\section{Materials and Methods}

Plant material. 'Jewel,' 'Sumor,' and L87-95 storage roots were used. A single sprout from a bedded root of each genotype served as source of nodal explants for in vitro culture on a basal MS medium (Murashige and Skoog, 1962). The remaining portion of each sprout was grown in the greenhouse and eventually transplanted to field beds in 1991. Thereafter, the latter material was subjected to additional cycles (one cycle = planting, root harvest, sprout production) of conventional adventitious propagation using sprouts that arise from storage roots. Seven propagation cycles were achieved by alternating field culture with a greenhouse culture phase over the winter. All U.S. no. 1 grade storage roots per genotype per cycle were retained. On the other hand, in vitro shoot cultures were serially subcultured for seven passages on the basal MS medium to approximate the generation time for the conventional adventitious-propagated plans. For each passage, about 5$7 \mathrm{~mm}$ long stem sections containing a single node were subcultured 
on to the culture medium. At least 50 plantlets were retained per genotype with each passage. Before field plantings, all in vitro plantlets were moved to the greenhouse for hardening.

In 1994, plant materials from all the propagation methods were simultaneously grown in field beds using a randomized complete block design. Following 8 weeks of growth, leaves were sampled from 10-20 plants selected at random from each genotype-propagation treatment. The first 2-5 fully opened leaves were collected from sample vines of each plant, bulked, freeze-dried, and stored at $-80 \mathrm{C}$ before DNA extraction. In this study, 'Jewel,' 'Sumor,' and L87-95 adventitious materials were arbitrarily designated by $\mathrm{JF}, \mathrm{SF}$, and $95 \mathrm{~F}$, followed by a provisional sample number. Likewise, nodally propagated materials were designated JT, ST, and 95T, also followed by a provisional sample number.

DNA extraction. Total DNA was isolated based on the method of Saghai-Maroof et al. (1984) as modified by Jarrett and Austin (1994). Total DNA was extracted from $0.3 \mathrm{~g}$ of bulked freeze-dried leaf tissue from each of 10 sample plants per genotype-propagation treatment. Freeze-dried leaf tissue was ground to a fine powder in liquid nitrogen. An isolation buffer $(50 \mathrm{~mm}$ Tris/ $\mathrm{HCl} \mathrm{pH}$ 8.0, $25 \mathrm{~mm}$ EDTA pH 8.0, $0.35 \mathrm{~mm}$ sorbitol, 5\% (PVP-40) polyvinylpyrolidone, $1 \%$ sodium bisulfite, and $0.2 \%$ of $2-$ mercaptoethanol) was added to the powder and centrifuged at $2000 \times g$ for $10 \mathrm{~min}$ at $4 \mathrm{C}$. The supernatant and all loose debris were discarded. The pellet (crude nuclei) was resuspended in extraction buffer $\left(5 \mathrm{ml} \cdot \mathrm{g}^{-1}\right.$ fresh weight) $(100 \mathrm{~mm}$ Tris $/ \mathrm{HCl} \mathrm{pH} \mathrm{8.0,1.4} \mathrm{M}$ $\mathrm{NaCl}, 20$ mm EDTA, 2\% hexadecyltrimethylammonium bromide, and $1 \%$ of 2-mercaptoethanol) followed by incubation at $60 \mathrm{C}$ for $30-60 \mathrm{~min}$. An equal volume of 24 chloroform : 1 isoamyl alcohol (v/v) was used for extraction for 5 min with slow but constant inversion, and phases were separated by centrifugation at $5,000 \times$ $g$ for $10 \mathrm{~min}$ at room temperature. The aqueous phase was removed and transferred to a new tube. DNA was further reextracted with 24 chloroform : 1 isoamyl alcohol (v/v) and transferred to a new tube for precipitation. About 2/3 volume of isopropanol was added, and the tube was inverted quickly several times until the DNA was precipitated and then removed. All DNA samples were dissolved in $10 \mathrm{~mm}$ Tris- $\mathrm{HCl}, \mathrm{pH} 7.5$., and $1 \mathrm{~mm}$ EDTA, and then quantified using a spectrophotomer (UV-VIS; Perkin-Elmer, Norwalk, Conn.)

Arbitrary primers and amplification conditions. Forty decamer oligonucleotide primers from kits A and F (Operon Technologies, Norwalk, Conn.) were evaluated for suitability of amplification in prior studies (Villordon and LaBonte, 1995). Fifteen primers were selected in the final study including OPA-01, OPA-07, OPA-09, OPA-10, OPA-11, OPA-15, OPA-19, OPF-01, OPF-05, OPF-06, OPF-08, OPF-12, OPF-14, OPF-16, and OPF-17. These primers were selected based on the ability to produce at least one putative polymorphic marker locus and reproducibility of the marker fragments. Genomic DNA of 10 sample plants randomly selected from each genotype-propagation treatment $(n=60)$ was used in amplification assays. Two separate replicate runs were done for each primer-treatment combination.

GeneAmp PCR reagent kits (Perkin-Elmer Applied Biosystems, Foster City, Calif.) were used in all reaction mixtures. Reaction conditions were similar to those reported by Williams et al. (1990), except that we used 1.5 units of Taq Stoffel fragment (PerkinElmer Applied Biosystems). PCR conditions consisted of $10 \mathrm{~mm}$ Tris- $\mathrm{HCl}, \mathrm{pH} 8.2,50 \mathrm{~mm} \mathrm{KCl}, 2 \mathrm{~mm} \mathrm{MgCl}_{2}, 0.001 \%$ gelatin, 0.1 mM each of dATP, dCTP, dTTP, dGTP, 0.2 mm primer, and $20 \mathrm{ng}$ genomic DNA per $25 \mu \mathrm{l}$ reaction volume. The reaction mixture was placed in a $0.5 \mathrm{ml}$ plastic reaction tube and overlaid with $25 \mu \mathrm{l}$ of sterile mineral oil. DNA was amplified in a thermal cycler (model 480; Perkin-Elmer) programmed for 40 cycles of $1 \mathrm{~min}$ at
94C, $1 \mathrm{~min}$ at 35C, $2 \mathrm{~min}$ at 72C, followed by a final extension at $72 \mathrm{C}$ for $7 \mathrm{~min}$, and then held at $4 \mathrm{C}$ until recovery. Each amplification included control, reaction mixtures that did not contain template DNA. Amplified DNA fragments were resolved by electrophoresis $(3 \mathrm{v} / \mathrm{cm})$ for $3 \mathrm{~h}$ and $20 \mathrm{~min}$ on $1.2 \%$ agarose (BRL, Bethesda, Md.) gels in $1 \times$ TAE buffer $(0.04 \mathrm{M}$ Tris-acetate and $0.001 \mathrm{~m}$ EDTA) in the presence of $0.5 \mu \mathrm{g} / \mathrm{ml}$ ethidium bromide. These were photographed using UV light. A 1-kb DNA ladder (BRL) was used as a molecular weight marker. The presence or absence of bands between 200 and $1000 \mathrm{bp}$ were scored from photographs.

Analysis of amplified fragments. After excluding markers that were monomorphic for the entire data set, a vector of molecular marker phenotype was established for each individual plant analyzed. Variability was expressed as percent polymorphism computed as the number of polymorphic markers over the total number of scored markers. In addition, fragment data were treated as twostate qualitative data from which pairwise genetic similarity/ dissimilarity measurements were estimated. For genetic similarity estimates, band data were coded as one (presence of band) and zero (absence of band), and entered into a Numerical Taxonomy and Multivariate Analysis System (NTSYS-pc), version 1.8 (Applied Biostatistics, Setauket, N.Y.) Genetic similarity was estimated using Similarity for Qualitative Data (SIMQUAL = Dice option) to generate Dice's similarity coefficient in NTSYS-pc (Applied Biostatistics). A triangular matrix of similarity values was generated and subjected to principal components analysis and multidimensional scaling (MDSCALE) algorithm (NTSYS-pc). On the other hand, genetic distance was estimated using the Euclidean metric of Excoffier et al. (1992) defined here as

$$
E=\left\{\varepsilon_{\text {xy }}^{2}\right\}=\mathrm{n}\left[1-2 \mathrm{n}_{\mathrm{xy}} / 2 \mathrm{n}\right]
$$

where $2 \mathrm{n}_{\mathrm{xy}}$ is the number of markers shared by two individuals, and $\mathrm{n}$ is the total number of polymorphic sites. This measure amounts to a tally of band differences between individuals (Huff et al., 1993) and creates a pairwise genetic distance matrix among all samples. This matrix was subjected to the Analysis of Molecular Variance (WINAMOVA 1.53) (Excoffier 1993; Excoffier et al., 1992; Huff et al., 1993) to estimate variance components attributed to among-genotype and within-genotype (propagation) effects. The AMOVA converts the intra-individual distance matrix into an equivalent analysis of variance. A classic variance-components extraction yields the variance components of interest. Significance level for variance components estimates are computed through nonparametric permutational procedures (Huff et al., 1993).

\section{Results}

Marker profile. The sample of 15 random sequence oligonucleotide primers generated 64 scorable markers among the sample set $(n=60)$ with an average of 3.87 markers per primer. Of the 64 scorable fragments, 29 were scored as putatively polymorphic marker loci (Table 1, Fig. 1). The number of polymorphic markers scored for each primer, assuming that each amplified product was from a unique locus, varied from one (A01, A09, F01, F-12, F-14, $\mathrm{F}-17$ ) to three (A-07, A-10, F-08, F-16), with an average of 1.87 per primer. Across the entire sample set, 19 marker loci were associated with putative fixed differences that were diagnostic to cultivar, 17 of which were also putatively polymorphic within cultivar, while two were monomorphic within cultivar. The remaining nine marker loci represented fragments that were shared by all genotypic samples. All nine (14.1\%) loci were polymorphic within 
Table 1. Attributes of 15 oligonucleotide primers used for generating arbitrarily primed markers among 60 sweetpotato clones representing three genotypes and two $(\mathrm{F}=$ adventitious, $\mathrm{T}=$ nodal $)$ propagation methods.

\begin{tabular}{|c|c|c|c|c|c|c|c|c|c|}
\hline \multirow[b]{4}{*}{ Primer } & \multirow{4}{*}{$\begin{array}{l}\text { No. of } \\
\text { markers } \\
\text { scored }^{\mathrm{x}}\end{array}$} & \multirow{4}{*}{$\begin{array}{c}\text { Putatively } \\
\text { polymorphic } \\
\text { markers (bp) }^{\mathrm{w}}\end{array}$} & \multicolumn{7}{|c|}{ No. of putative polymorphisms ${ }^{z}$} \\
\hline & & & \multirow{3}{*}{$\begin{array}{c}\text { Among } \\
\text { genotypes }^{v}\end{array}$} & \multicolumn{6}{|c|}{ Within genotypes/among propagation ${ }^{y}$} \\
\hline & & & & \multicolumn{2}{|c|}{ Jewel } & \multicolumn{2}{|c|}{ Sumor } & \multicolumn{2}{|c|}{ L87-95 } \\
\hline & & & & $\mathrm{F}$ & $\overline{\mathrm{T}}$ & $\bar{F}$ & $\overline{\mathrm{T}}$ & $\mathrm{F}$ & $\mathrm{T}$ \\
\hline$\overline{\mathrm{OPA}-01}$ & 2 & _344 & 1 & 0 & 0 & 1 & 0 & 1 & 0 \\
\hline OPA-07 & 4 & _344_451_506 & 2 & 0 & 0 & 0 & 0 & 3 & 0 \\
\hline OPA-09 & 5 & _396_890 & 1 & 0 & 0 & 0 & 0 & 1 & 0 \\
\hline OPA-10 & 7 & _298_344_890 & 1 & 0 & 0 & 1 & 0 & 3 & 0 \\
\hline OPA-11 & 4 & _506_634 & 1 & 0 & 1 & 1 & 0 & 2 & 0 \\
\hline OPA-15 & 3 & _762_890 & 1 & 0 & 0 & 2 & 0 & 1 & 0 \\
\hline OPA-19 & 5 & _634_1018 & 2 & 1 & 1 & 0 & 0 & 0 & 0 \\
\hline OPF-01 & 5 & -762 & 1 & 0 & 0 & 1 & 0 & 1 & 0 \\
\hline OPF-05 & 4 & _506_634 & 2 & 0 & 0 & 0 & 0 & 2 & 0 \\
\hline OPF-06 & 3 & _506_634 & 1 & 1 & 0 & 1 & 0 & 1 & 0 \\
\hline OPF-08 & 5 & _451_540_890 & 2 & 1 & 0 & 1 & 0 & 2 & 0 \\
\hline OPF-12 & 2 & _396 & 1 & 0 & 0 & 0 & 0 & 0 & 1 \\
\hline OPF-14 & 1 & -762 & 0 & 0 & 0 & 0 & 0 & 1 & 0 \\
\hline OPF-16 & 6 & _298_540_762 & 2 & 0 & 0 & 1 & 0 & 2 & 0 \\
\hline OPF-17 & 8 & _344 & 1 & 0 & 0 & 0 & 0 & 0 & 0 \\
\hline Total & 64 & 29 & 19 & 3 & 2 & 9 & 0 & 20 & 1 \\
\hline
\end{tabular}

${ }^{\mathrm{z} V a l u e s}$ in parentheses estimate extent of polymorphism computed as number of putative polymorphic loci divided by the total number of scored fragments.

${ }^{\mathrm{y}}$ Include scored amplified fragments that are shared by all samples as well as marker loci that are diagnostic to genotypic class.

${ }^{\mathrm{x}}$ Scored marker loci that are diagnostic to genotypic class and exclude fragments that are shared across samples.

"Markers are designated by "_" followed by approximate fragment size in base pairs. Multiple fragments are represented by a string of " " plus fragment size in bp. Fragment size was based on visual comparison with molecular weight marker (1-kb DNA ladder, BRL, Bethesda, Md.)

v'Scored marker loci that are diagnostic to genotypic class and exclude fragments that are shared across samples.

adventitiously derived plants whereas only three $(4.7 \%)$ were polymorphic within the nodally derived plants.

In general, there was comparatively greater genomic uniformity (fewer marker polymorphisms) within the nodally derived materials. Among the nodally derived lineages, putative polymorphisms were $3.1 \%, 0.0 \%$, and $1.6 \%$ for JT, ST, and 95T, respectively. In contrast, putative polymorphisms within adventitiously derived JF, SF, and 95F plants were $4.7 \%, 14.1 \%$, and $31.3 \%$. At the fourth generation, a white root skin and flesh color mutation was detected among the 'Jewel' adventitious materials. The phenotypic mutant was included in the MDSCALE analysis for comparative purposes but was excluded in the "analysis of molecular variance' so as not to skew the results.

Genetic similarity. The $60 \times 60$ matrix of genetic similarity measures (data not shown) was subjected to common principal component analysis as well as multidimensional scaling (MDSCALE, NTSYS-pc; Applied Biostatistics). The first and second principal components accounted for $75 \%$ and $8 \%$ of the variance, respectively. MDSCALE is related to principal components analysis, but fits an appropriate model in the fewest dimensions possible. The principal coordinate scatter plots for the MDSCALE analysis (number of dimensions in configuration space $=2$ ) is presented using the MXPLOT graphing program in the NTSYS-pc software (Fig. 2). The data set was analyzed with and without the mutant 'Jewel' (Jmu) adventitious sample. In both analyses, three genotypes were differentiated along with putatively variant samples. The MDSCALE analysis that includes 'Jmu' is presented.

The MDSCALE plot shows a clear separation between geno-

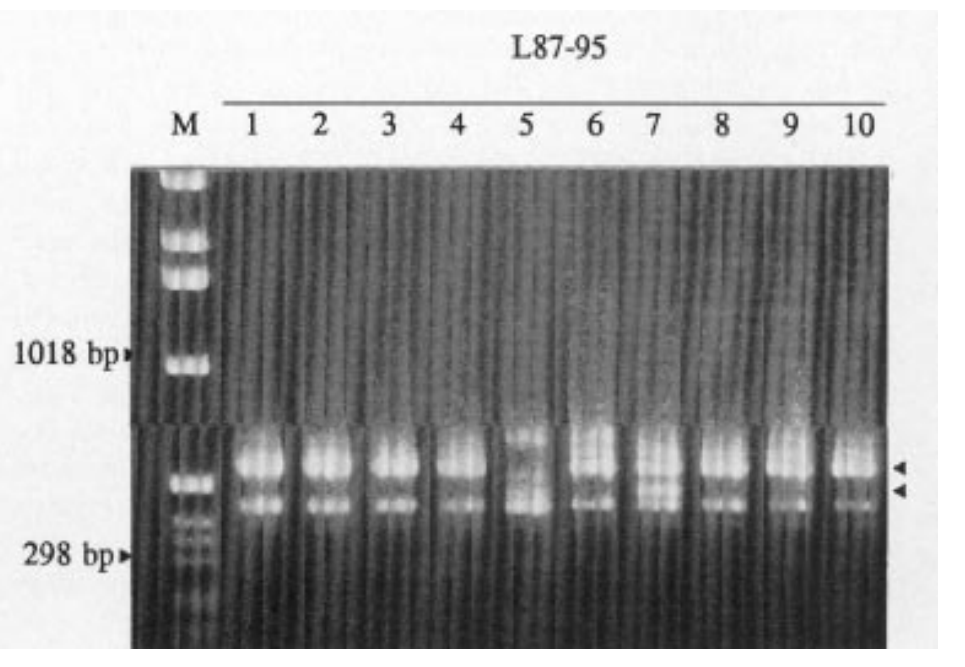

Fig. 1. Ethidium bromide-stained amplification products from genomic DNA of 10 L87-95 sweetpotato adventitiously derived clones using primer OPF-05. Lane M $=1-\mathrm{kb}$ DNA ladder. Putative polymorphic loci are indicated by left-facing arrowheads.

types 'Jewel', 'Sumor', and L87-95. The plot also shows distribution of putative polymorphic phenotypes: JF7, JF9, JT10, 95F6, 95F7, 95F8, 95T7, 95T10, SF6, and Jmu.

Propagation effects. A $60 \times 60$ matrix of Euclidean distances was entered into the AMOVA 1.53 program and variance components were estimated. The majority of the total genetic variability (putative marker polymorphisms) was attributed to genotypic 


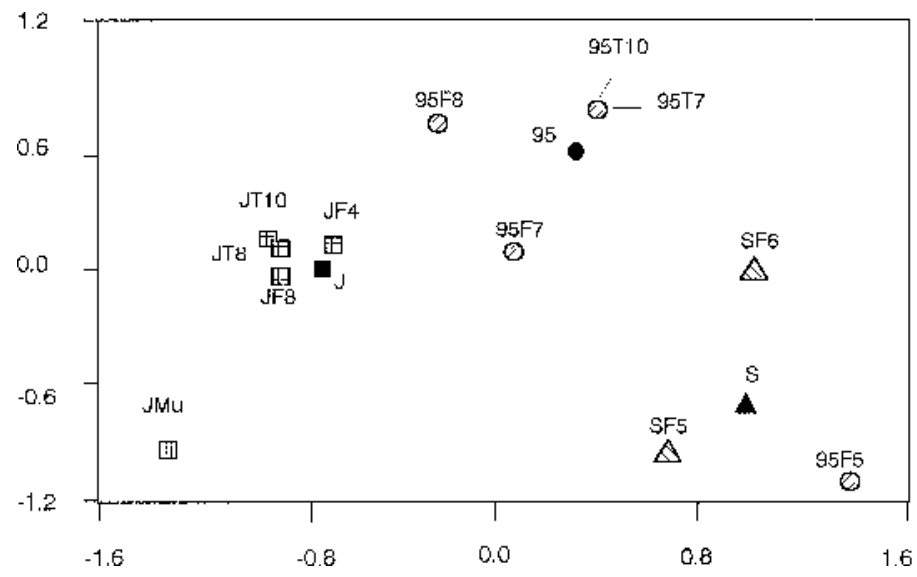

effects $(88.70 \%, P<0.001)$ (Table 2). However, the 'within genotype' component (propagation effects) accounted for $11.38 \%$ of total variability $(P=0.003)$. In addition to differentiating genotypes, markers identified 11 putative variants relative to majority of marker phenotypes that characterized the respective genotypic classes. Among putatively variant samples for marker phenotypes, seven $(23 \%)$ were from adventitiously derived materials, whereas four $(13.33 \%)$ were derived from nodal plants. Putative polymorphisms were detected only among 'Jewel' and L87-95 lineages that were nodally propagated; none were detected among Sumor nodal samples.

\section{Discussion}

Arbitrarily primed molecular markers. The sample of random primers generated numerous marker loci that differentiated among genotypes and putative variant marker phenotypes among samples used in this study. The genotypic effect accounted for a significantly large variance component estimate $(P<0.001)$. Despite the relatively large genotypic variability, the AMOVA procedure detected a comparatively smaller 'within genotype' (propagation) effect $(P=0.003)$. As all clones within each genotype are derived from a single sprout, this within genotype variability (putative marker polymorphisms ) can only be attributed to propagation effects.

Molecular marker data provide an assay of genomic uniformity that is generally independent of confounding effects of the environment. However, the magnitude of molecular polymorphisms does not necessarily reflect the degree of phenotypic variability (De Klerk, 1990). Except for the fleshy root skin and flesh color variation that was observed in the fourth-generation 'Jewel' adventitious materials, other highly visible phenotypic changes were absent among root or vine traits. On occasion, molecular markers can be directly correlated
Fig. 2. Plot of first and second dimensions of MDSCALE analysis of the genetic similarity matrix of 60 samples representing three sweetpotato genotypes propagated through adventitious and nodal methods. Designation for data points is defined by genotype followed by provisional sample serial number. JF = Jewel, adventitious; $\mathrm{SF}=$ Sumor, adventitious; 95F = L87-95 adventitious; JT = Jewel, nodal; ST = Sumor, nodal; and, 95T $=$ L87-95 nodal. Solid figures $(\boldsymbol{\square}, \mathbf{A}, \mathbf{O})$ represent multiple data points representing the majority of samples with monomorphic fragment patterns that are diagnostic to each genotype. Jmu = Jewel white root skin and flesh color mutation detected at generation four.

with phenotypic variability. For instance, restriction fragment length polymorphisms (RFLPs) were associated with mutated chloroplasts in albino potato plants regenerated from tissue culture (Day and Ellis, 1985). In contrast, even a $75 \%$ reduction of $25 \mathrm{~S}$ ribosomal DNA does not phenotypically differentiate mutants from wild type plants. Moreover, in phylogenetic studies, descent trees based on molecular data do not necessarily correlate with morphological data (De Klerk, 1990). Molecular markers can only be directly correlated with qualitatively and quantitatively inherited traits through analysis of polymorphisms in plant populations segregating for a phenotypic trait of interest.

Arbitrarily primed markers sample the whole genome, ranging from conserved, functionally important sequences, to middle-, and highly repetitive sequences (Williams et al., 1990). In plants, highly repetitive sequences range from $25 \%$ (Leutwiler et al., 1984) to $80 \%$ (Flavell, 1985) of the total genomic content. In this study, it is possible that many primers detected sequence changes and gross chromosomal mutations associated with middle- and highly repetitive sequences. While the precise functional significance of the repetitive sequences is still unclear, it is generally agreed that these redundant sequences are mainly responsible for the wide variation of genome size among higher plants (Bennett et al., 1982). Moreover, repetitive DNA sequences associated with centromeres, telomeres, constitutively heterochromatic regions, and nucleolar organizing regions in mammalian cells appear to provide a structural framework for nuclear events that include gene transcription and silencing (Haaf and Schmid, 1991). Thus, evidence indicates that many repetitive gene sequences are not merely selfish or junk sequences, and can have direct effects at the phenotypic level. Somatic cell changes involving repetitive sequences as well as highly conserved base sequences that have direct functional significance can be fixed through random drift especially in small populations in the asexually propagated sweetpotato. These changes represent alterations to the original genomic constitution that characterized the cultivar and will eventually result in clonal variability and decline.

Nodal culture reduces genomic variability in the sweetpotato. Our results support the premise that preformed meristematic cells, such as those found in sweetpotato nodes, give rise to plants that are more genetically stable (Potter and Jones, 1991). Meristematic

Table 2. Analysis of molecular variance (AMOVA; Excoffier, 1993) using 28 arbitrarily primed molecular markers for 60 sweetpotato samples representing three genotypes and two propagation methods.

\begin{tabular}{lcccccc}
\hline \hline $\begin{array}{l}\text { Source of } \\
\text { variation }\end{array}$ & $\mathrm{df}$ & $\mathrm{SSD}^{\mathrm{z}}$ & $\mathrm{MSD}^{\mathrm{y}}$ & $\begin{array}{c}\text { Variance } \\
\text { component }\end{array}$ & $\%$ Total $^{\mathrm{x}}$ & $P$ value $^{\mathrm{w}}$ \\
\hline $\begin{array}{l}\text { Among genotypes } \\
\begin{array}{l}\text { Within genotypes/ } \\
\text { among propagation }\end{array}\end{array}$ & 2 & 225.85 & 112.925 & 5.611 & $88.70 \%$ & $<0.001$ \\
\hline
\end{tabular}

${ }^{\mathrm{z}}$ Sums of squared deviations.

${ }^{\mathrm{y}}$ Mean squared deviations.

xPercent of total (6.326).

wProbability of obtaining a more extreme component estimate by chance alone. Number of permutations in data set; $\mathrm{n}=1000$. 
tissues provide strict control of cell division processes and minimize, but do not preclude, the risks of genomic changes such as DNA duplication that leads to somatic polyploidy and other irregularities (Sree Ramulu, 1987). Relative to the majority of samples monomorphic for marker loci, the genetic variation (putative marker polymorphisms) observed among nodally propagated materials might represent spontaneous genomic mutations that occur regardless of propagation method. Polyploidy is generally associated with increased mutation rates (Mogie, 1992), and variant cells that occur within wild-type cells, i.e. chimeras, can be transmitted in the clonal lineage especially if these variants occur in growing points (Hartmann and Kester, 1983).

Conclusions. Based on these results, nodal in vitro propagation procedures reduce genomic variability associated with adventitious propagation. Incorporation of nodal culture procedures in existing foundation seed programs can reduce genomic changes through use of preformed meristematic regions. This propagation process will result in genetically uniform clones that have fewer qualitative mutations and quantitative variability at the phenotypic level. The nodal culture phase can be integrated as a step where foundation seed are initially maintained in culture, and serve as source of propagating material for each cycle of propagation. The in vitro culture step does not guarantee genomic purity, but can minimize inherent genomic variability.

\section{Literature Cited}

Applied Biostatistics. 1993. NTSYS-pc (numerical taxonomy and multivariate analysis system). Exeter Software, Setauket, N.Y.

Bennett, M.D., J.B. Smith, and J.S. Heslop-Harrison. 1982. Nuclear DNA amounts in angiosperms. Proc. Royal Soc. London Ser. B. 216:179-199.

Collins, W.W., L.G. Wilson, S. Arrendell, and L.F. Dickey. 1987. Genotype $\times$ environment interactions in sweetpotato yield and quality factors. J. Amer. Soc. Hort. Sci. 112:579-583.

Day, A. and T.H.N. Ellis. 1985. Deleted forms of plastid DNA in albino plants from cereal anther culture. Current Genet. 9:674-678.

De Klerk, G.J. 1990. How to measure somaclonal variation. Acta Bot. Neerl. 39:129-144.

Edmond, J.B. and G.R. Ammerman. 1971. Sweetpotatoes: Production, processing, and marketing. AVI, Westport, Conn.

Esau, K. 1977. Anatomy of seed plants. 2nd ed. Wiley, New York.

Excoffier L., P.E. Smouse, and J.M. Quattro (1992). Analysis of molecular variance inferred from metric distances among DNA haplotypes: Application to human mitochondrial DNA restriction sites. Genetics 131:479-491.

Fahn, A. 1982. Plant anatomy. Pergammon Press, Oxford.

Flavell, R.B. 1985. Repeated sequences and genome change, p. 129-156. In: B. Hohn and E.S. Dennis (eds.). Genetic flux in plants. Springer Verlag, Vienna.
Haaf, T. and M. Schmid. 1991. Chromosome topology in mammalian interphase nuclei. Expt. Cell. Res. 192:325-332.

Hartmann, H.T. and D.E. Kester. 1983. Plant propagation-Principles and practices. 4th ed. Prentice-Hall, Englewood Cliffs, N.J.

Huett, D.O. 1982. Evaluation of sources of propagating material for sweetpotato production. Sci. Hort. 16:1-7.

Huff, D.R., R. Peakall, and P.E. Smouse. 1993. RAPD variation within and among natural populations of outcrossing buffalograss [Buchloe dactyloides (Nutt.) Engelm.]. Theor. Appl. Genet. 86:927-934.

Jarrett, R.L. and D.F. Austin. 1994. Genetic diversity and systematic relationships in sweetpotato (Ipomoea batatas L. Lam) and related species as revealed by RAPD analysis. GRACE (In press.)

Kannua, M.B. and C.N. Floyd. 1988. Sweetpotato genotype-environment interactions in the highlands of Papua, New Guinea. Trop. Agr. 65:9-15.

Leutwiler, L.S., B.R. Hough-Evans, and E.M. Meyerowitz (1984). The DNA of Arabidopsis thaliana. Mol. General Genet. 194:15-23.

Mogie, M. 1992. Evolution of asexual reproduction in plants. Chapman and Hall, London.

Murashige, T. and F. Skoog. 1962. A revised medium for rapid growth and bioassays with tobacco tissue cultures. Physiol. Plant. 15:473-497.

Ngeve, J.M. and J.C. Bouwkamp. 1993. Comparison of statistical methods to assess yield stability in sweetpotato. J. Amer. Soc. Hort. Sci. 118:304-310.

Potter, R. and M.G.K. Jones. 1991. An assessment of genetic stability of potato in vitro by molecular and phenotypic analysis. Plant Sci. 76:239248.

Saghai-Maroof, M.A., K.M. Soliman, R.A. Jorgenson, and R.W. Allard. 1984. Ribosomal DNA spacer-length polymorphisms in barley: Mendelian inheritance, chromosomal location, and population dynamics. Proc. Natl. Acad. Sci. USA 81:8014-8018.

Sloan, Jr., R.C. 1994. Mississippi sweetpotato foundation seed program. HortTechnology 4:230-232.

Sree Ramulu. 1987. Genetic instability during plant regeneration in potato: Origin and implications. Plant Physiol. 6:211-218.

Steinbauer, C.E., G.P. Hoffman, and J.B. Edmond. 1943. Why are single plant yields of sweetpotato highly variable within plots? J. Amer. Soc. Hort. Sci. 43:249-254.

Templeton-Somers, K. and W.W. Collins. 1986. Field performance and clonal variability in sweetpotatoes propagated in vitro. J. Amer. Soc. Hort. Sci. 111:689-694.

Villordon, A.Q. and D.R. LaBonte. 1995. Variation in randomly amplified DNA markers and storage root yield in 'Jewel' sweetpotato clones. J. Amer. Soc. Hort. Sci. 120:734-740.

Welsh, J. and M. McClleland. 1990. Fingerprinting genomes using PCR with arbitrary primers. Nucleic Acids Res. 18:7213-7218.

Williams, J.G.K., A.R. Kubelic, K.J. Livak, J.A. Rafalski, and S.V. Tingey. 1990. DNA polymorphisms amplified by arbitrary primers are useful as genetic markers. Nucleic Acids Res. 18:6531-6535.

Yen, D.E. 1974. The sweetpotato and Oceania. Bishop Museum Bul., Honolulu 236:1-389. 